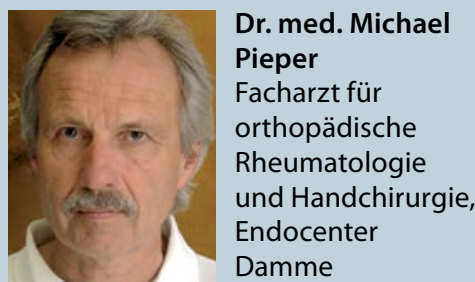

Biologikum und MTX gegenüber einer MTX-Monotherapie oder einer Kombinationstherapie mit anderen DMARDs.

Als problematisch erscheint der Zulassungsstatus, der bei Infliximab für die Behandlung der JIA nicht gegeben ist. Auch sollte beim Einsatz von Infliximab das Sicherheitsprofil in dieser Altersgruppe mit beachtet werden. In klinischen Studien wurden Infektionen bei einem höheren Anteil der Kinder und Jugendlichen berichtet als bei erwachsenen Patienten.

Nach der Markteinführung wurden maligne Erkrankungen, einige mit tödlichem Ausgang bei Kindern, Jugendlichen und jungen Erwachsenen (bis zu 22 Jahre alt) beschrieben, die mit TNF-Blockern - einschließlich Infliximab - behandelt wurden. Ungefähr die Hälfte der Fälle waren Lymphome. Ein Risiko für die Entwicklung maligner Erkrankungen bei Kindern und Jugendlichen, die mit TNF-Blockern, einschließlich Infliximab behandelt werden, kann also nicht ausgeschlossen werden. Insofern ist es umso wichtiger, Risiko und Nutzen einer solchen Therapie gegeneinander abzuwägen (Quelle: Fachinformation der Firma MSD über Remicade ${ }^{\circledast}$ ).

Tynjälä P et al. Aggressive Combination Drug Therapy in Very Early Polyarticular Juvenile Idiopathic Arthritis (ACUTE-JIA): a multicentre randomised open-label clinical trial. Ann Rheum Dis 2011; 70: 1605

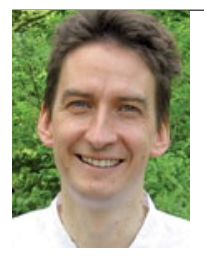

Dr. med. Marco

Semmler Klinikum Südstadt Rostock, Südring 81 18059 Rostock

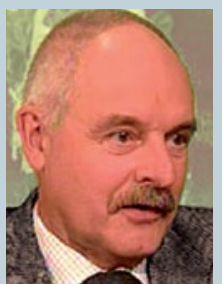

Prof. Dr. Klaus A. Milachowski Orthopädische Privatpraxis, Belegarzt an der Klinik Dr. Schreiber, München

\title{
Motorische Regeneration nach Schlaganfall: Elektrostimulation hilft langfristig
}

Eine neuromuskuläre Elektrostimulation kann die motorische Funktion der oberen Extremitäten sowie die Fähigkeit zu Alltagsaktivitäten nach einem Schlaganfall verbessern. Unklar war bislang, wie nachhaltig diese Erfolge sind.

E ine chinesische Studie hat jetzt unEtersucht, wie lang die erreichten Effekte der neuromuskulären Elektrostimulation (NES) nach dem Ende der Behandlung anhalten. 46 Schlaganfallpatienten wurden einer Kontrollgruppe oder einer NES-Gruppe zugeteilt. Zudem erhielten alle Patienten ein Standard-Rehabilitationsprogramm. Die NES erfolgte über drei Wochen an fünf Tagen die Woche für jeweils 30 Minuten. Beurteilt wurde der Zustand vor Beginn der Behandlungen, in der zweiten und dritten Behandlungswoche sowie ein, drei und sechs Monate nach dem Ende der Therapie. Für die Beurteilung der Spastik wurde die Modified Ashworth Scale (MAS) eingesetzt, mit dem Fugl-Meyer Assessment (FMA) wurde die motorische Beweglichkeit der oberen Extremität beurteilt, und der Modified Barthel-Index (MBI) spiegelte die allgemeinen Fähigkeiten des Patienten im täglichen Leben wider. 37 der 46 Patienten beendeten die Studie.

Nach der dritten Behandlungswoche konnten im Vergleich mit der Kontrollgruppe signifikante Verbesserungen sowohl bei der Beweglichkeit der oberen Extremitäten als auch hinsichtlich der Spastik nachgewiesen werden (FMA-Gesamt-Score 20,3 versus 14,5;
MAS 1,16 versus 0,78 ; MBI 57,0 versus 49,8). Diese Verbesserungen hielten auch einen Monat nach dem Ende der Behandlung an. Auch bei den Kontrolluntersuchungen drei und sechs Monate nach dem Ende der NES waren einige der Erfolge noch nachweisbar. Der durchschnittliche Score war in der NES-Gruppe noch immer signifikant besser als in der Kontrollgruppe (nach sechs Monaten FMA Gesamt-Score: 29,8 versus 20,3 ; MAS: 1,67 versus $1,86$; MBI 79,2 versus 66,1$)$.

Schlussfolgerung: Erhalten Patienten nach einem Schlaganfall eine dreiwöchige neuromotorische Elektrostimulation, erholen sich ihre motorischen Fähigkeiten schneller. Dieser Vorteil bleibt mindestens sechs Monate nach Ende der Behandlung erhalten. Dr. Christine Starostzik

Ziling Lin et al. Long-term effectiveness of neuromuscular electrical stimulation for promoting motor recovery of the upper extremity after stroke. J Rehabil Med 2011;43:504-510 